\title{
Performance analysis of the differential pulse-width pair Brillouin optical time domain analysis using the log normalized and linearly normalized gain
}

H. D. Bhatta, S. A. Zaslawski, Z. Yang, M. Tur, L. Thévenaz

H. D. Bhatta, S. A. Zaslawski, Z. Yang, M. Tur, L. Thévenaz, "Performance analysis of the differential pulse-width pair Brillouin optical time domain analysis using the log normalized and linearly normalized gain," Proc. SPIE 11199, Seventh European Workshop on Optical Fibre Sensors, 111992J (28 August 2019); doi: 10.1117/12.2541352

Event: Seventh European Workshop on Optical Fibre Sensors, 2019, Limassol, Cyprus 


\title{
Performance analysis of the differential pulse-width pair Brillouin optical time domain analysis using the log normalized and linearly normalized gain
}

\author{
H.D. Bhatta*a,b, S. A. Zaslawski ${ }^{\mathrm{b}}$, Z.Yang ${ }^{\mathrm{b}}$, M. Tur ${ }^{\mathrm{a}}$, L. Thévenaz ${ }^{\mathrm{b}}$ \\ ${ }^{a}$ School of Electrical Engineering, Tel-Aviv University, Tel-Aviv, 6997801, Israel; ' ${ }^{\mathrm{b}}$ Institute of \\ Electrical Engineering, EPFL Swiss Federal Institute of Technology, SCI STI LT, Station 11, CH- \\ 1015 Lausanne, Switzerland
}

\begin{abstract}
The performance of the differential pulse-width pair Brillouin optical time domain analysis (DPP-BOTDA) is evaluated experimentally using either the gain from log normalization or linear normalization for the subtraction of traces collected with pump pulses of slightly different pulse widths. Using pump pulses widths of $43 \mathrm{~ns}$ and $40 \mathrm{~ns}$, amplified Brillouin time domain probe traces were obtained for $10 \mathrm{~km}$ of standard single mode fiber. Two hotspots of length $30 \mathrm{~cm}$ and $6 \mathrm{~m}$, separated by more than the spatial resolutions of the individual pulses and kept in a temperature controlled hot bath facility, were interrogated with temperature variations from 5 to $70{ }^{\circ} \mathrm{C}$, having probe signal gain of $\sim 40 \%$ at the Brillouin Frequency Shift (BFS). This research work demonstrates, for the first time, that the use of linear gains for the subtraction step in creating the Brillouin gain spectrum, produces results for small to medium Brillouin frequency shifts $(\leq 30 \mathrm{MHz}$ ), that deviate from the results of the subtraction of the logarithmic gains by as much as $2 \mathrm{MHz}$ $\left(\sim 2^{\circ} \mathrm{C}\right)$, particularly for hotspots of the order of the spatial resolution of the DPP-BOTDA. For hotspots longer than the spatial resolution of the technique, the difference between results of the two processing methods show BFS deviations only at the end of the hotspots.
\end{abstract}

Keywords: Brillouin Frequency Shift, DPP-BOTDA, spatial resolution, measurand accuracy, BFS deviation

\section{INTRODUCTION}

Distributed fiber-optic sensors employing Brillouin Optical Time Domain Analysis (BOTDA) are being used for the measurement of temperature and strain, due to their robust, linear and stable characteristics under different environmental conditions. ${ }^{1,2}$ In these interrogators, competing parameters are the signal to noise ratio (SNR), sensing speed, sensing range, dynamic measurand (e.g. temperature, strain) range, spatial resolution and measurand resolution, which need to be optimized. ${ }^{3,5}$ Spatial resolution, which is limited by the width of probing pump pulse, is very important for many applications. In Structural Health Monitoring (SHM), for example, centimeter-scale measurements are high in demand, where cracks or damages in critical structures can be identified early on and losses can be prevented. The high measurand accuracy in standard BOTDA can be achieved under the conditions of dense frequency sampling, availability of high SNR and narrowness of the Brillouin Gain Spectrum (BGS), which in turn may depend on the other interrogation parameters (e.g. pump power, length of the fiber, pump pulse width, etc.).$^{6,7}$ The width of the BGS is related to the phonon lifetime in the fiber and has a value of $\sim 30 \mathrm{MHz}$ in standard single mode fibers at around $1550 \mathrm{~nm}$. While the spatial resolution of a standard BOTDA configuration is determined by the width of the pump pulse, reducing the latter to below $\sim 40 \mathrm{~ns}$ to improve spatial resolution, comes at the expense of widening the BGS, thereby significantly lowering the measurand resolution. Among various schemes that have been used in the past to enhance the spatial resolution, Differential Pulsewidth Pair Brillouin Optical Time Domain Analysis is a quite efficient one. ${ }^{8}$ In DPP-BOTDA, two long pump pulses of slightly different widths are launched into the fiber (against a CW probe), and the two Brillouin responses of the fiber are manipulated, normally by subtracting their Brillouin 'gains' (see below) to obtain spatial resolution equivalent to that of a pump pulse as thin as the difference between the widths of the two pump pulses, while maintaining the narrowness of the BGS. In this 'gain' subtraction, two types of gains have been practiced: The linear gain: (Probe_out_powerProbe_input_power)/Probe_input_power and the logarithmic gain, which is defined by $\log$ (Probe_out_power/Probe_input_power). To ensure a good SNR, large Brillouin gains (>20\%) are usually targeted when implementing DPP-BOTDA. In this case, extracting the Brillouin Frequency Shift (BFS) from the gain difference may

Seventh European Workshop on Optical Fibre Sensors, edited by Kyriacos Kalli, Gilberto Brambilla, Sinead O'Keeffe Proc. of SPIE Vol. 11199, 111992J · @ 2019 SPIE · CCC code: 0277-786X/19/\$21 · doi: 10.1117/12.2541352 
depend on the type of gain used. In a previous work on pulse coding ${ }^{3}$, it has been found necessary to use the logarithmic gain for proper decoding.

In this article, we study the performance of the DPP-BOTDA technique as a function of these two types of gain, the linear and logarithmic ones. Experimental results show that subtracting the linear gains is the less accurate approach, giving rise to errors as much as $2 \mathrm{MHz}$ for the BFS. Below we study these two type of gains using 40 and $43 \mathrm{~ns}$ pump pulses while measuring two spatially separated hotspots of $30 \mathrm{~cm}$ and $6 \mathrm{~m}$, whose temperature was varied from 5 to $70^{\circ} \mathrm{C}$. The Brillouin gain was $\sim 40 \%$.

\section{LOG AND LINEAR NORMALIZATION SCHEMES FOR GAIN}

In the stimulated Brillouin amplification process, $\mathrm{CW}$ input probe power, $P_{i n, p r}(v, t)$, gets amplified by the counter propagating pump pulse power $P$ and width $T$ so as to generate amplified probe power, $P_{\text {out }, p r}(v, t)$, which is given by (neglecting pump depletion) : ${ }^{9,10}$

$$
P_{\text {out }, p r}(v, t)=P_{\text {in,pr }}(v, t) \exp \left[g\left(v-v_{B}\right) P L\right]
$$

Here, $g\left(v-v_{B}\right)$ is the Brillouin gain (in units of 1/[Watt-m]), having a narrow linewidth of $\sim 30 \mathrm{MHz}$ for pulse widths exceeding $40 \mathrm{~ns}, v \equiv v_{\text {probe }}-v_{\text {pump }}, v_{B}$ is local BFS, and $L=V_{g} T / 2$ is the interaction length (i.e., spatial resolution), where $V_{g}$ is the group velocity. The overall $\log$ and linear gains from a fiber segment of length (equivalent to spatial resolution), located at coordinate $z=V_{g} t / 2$ along the fiber-under-test (FUT) are defines as:

$$
G_{\text {log }}(v, \mathrm{z})=\log \left[\left(P_{\text {out }, p r}(v, z) / P_{\text {in }, p r}\right]=g\left(v-v_{B}(z)\right) P L,\right.
$$

and

$$
G_{\text {linear }}(v, z)=\left[P_{\text {out }, p r}(v, z)-P_{\text {in }, p r}\right] / P_{\text {in }, p r}=\exp \left[G_{\text {log }}(v, z)\right]-1
$$

As mentioned above, in DPP-BOTDA, normally two consecutive Brillouin traces, $P_{o u t, p r, T 1, T 2}(v, t)$ are collected from two pump pulses of slightly different widths. The results have been processed in the literature in two different ways to obtain the sought-for high spatial resolution trace. In the logarithmic approach the High Resolution Trace is given by:

$$
H R T_{\log }(\nu, \mathrm{z})=G_{\log , T 1}(\nu, \mathrm{z})-G_{\log , T 2}(\nu, \mathrm{z}),
$$

while linear processing produces:

$$
H R T_{\text {linear }}(v, \mathrm{z})=\left[P_{\text {out }, p r, T 1}(v, z)-P_{\text {out }, p r, T 2}(v, z)\right] / P_{\text {in,pr }}=\exp \left[G_{\text {log,T1 }}(v, z)\right]-\exp \left[G_{\text {log,T2 }}(v, z)\right] .
$$

Using Taylor series:

$$
H R T_{\text {linear }}(v, \mathrm{z}) \cong H R T_{\log }(v, \mathrm{z})+G_{\text {log }, T 1}(v, z)^{2} / 2-G_{\text {log, } T 2}(v, z)^{2} / 2,
$$

Since the high spatial resolution comes at the expense of SNR, strong pump pulses are launched, resulting in high Brillouin gains, thereby increasing the difference between the log and linear estimates. It is this difference that this paper is concentrating on.

\section{EXPERIMENTAL SETUP AND METHOD}

The Figure 1 is a typical standard BOTDA setup. The interrogating scanning frequency difference between pulsed pump and counter propagating continuous probe waves was varied between 10.5 to $10.65 \mathrm{GHz}$ with $1 \mathrm{MHz}$ scan step and 150 $\mathrm{MHz}$ scan width. The $10 \mathrm{~km}$ standard single mode fiber was interrogated at pulse repetition rate of $2.5 \mathrm{KHz}$, with pump pulses of 43 and $40 \mathrm{~ns}$. The two sections of interest, a $30 \mathrm{~cm}$ and $6 \mathrm{~m}$ long segments (spatially separated $>15 \mathrm{~m}$ ), both were emerged into hot bath with a precisely controlled temperature. The hot bath temperature was varied from 5 to $70{ }^{\circ} \mathrm{C}$ and the Brillouin probe gain traces were captured using a high sampling rate $(2.5 \mathrm{GHz})$ oscilloscope after passing via $800 \mathrm{MHz}$ band-width photo diode. Each trace was averaged 256 times for each polarization state where both of the polarizations 
reduce the polarization fading problem. For the chosen pump pulse widths the expected DPP-BOTDA resolution is $30 \mathrm{~cm}$ $\left(=2 \cdot 10^{8} \mathrm{~m} / \mathrm{s} \cdot(43-40) \mathrm{ns} / 2\right)$.

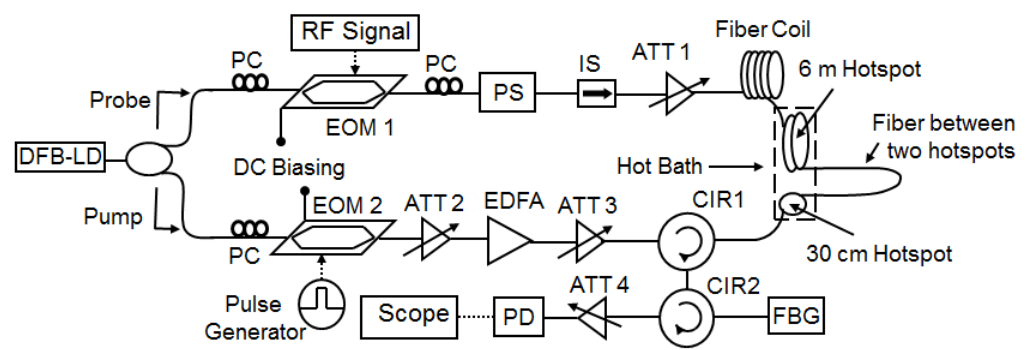

Figure 1. Experimental setup for the DPP-BOTDA: - DFB-LD: Distributed feedback laser diode; PC: polarization controller; PS: polarization switch; IS: isolator; ATT: Attenuator; FUT: fiber under test; EOM: electro optic modulator; FBG: Fiber Bragg grating filter; PD: photo diode; EDFA: Erbium-doped fiber amplifier; RF: Radio frequency.

\section{EXPERIMENTAL RESULTS}

While the two processing methods report identical BFS values for single pulse interrogation (verified but not shown), they differ when applied to the DPP-BOTDA technique and their difference depends on the size of the hotspots and interrogated temperature differentials. Figure 2 shows the BFSs results using DPP-BOTDA along the $30 \mathrm{~cm}$ (left) and $6 \mathrm{~m}$ (right) hotspots as the temperature varies between 5 to $70{ }^{\circ} \mathrm{C}$. The BFS curves for log and linear normalization are now different:
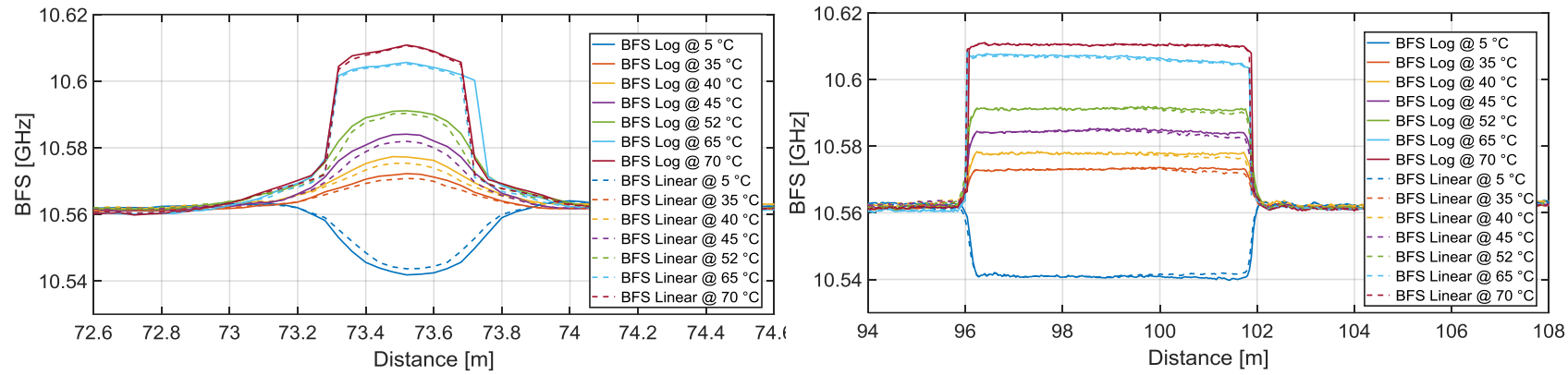

Figure 2. Left: The BFS along the $30 \mathrm{~cm}$ hotspot using the log (solid) and linear (dashed) normalizations, exhibiting a BFS difference as big as $2 \mathrm{MHz}$; Right: The BFS around the $6 \mathrm{~m}$ hotspot where results of the two methods appear to be much closer together with small deviation towards the end of segment, see Figure 3.

For the $30 \mathrm{~cm}$ hotspot, the observed deviation ranges from a maximum of $2 \mathrm{MHz}$ for low temperature differentials to convergence at their highest values. For the $6 \mathrm{~m}$ hotspot, only slight BFS deviations are observed $(<<1 \mathrm{MHz})$ towards the end of the heated segment, and, again, convergence at high temperatures.
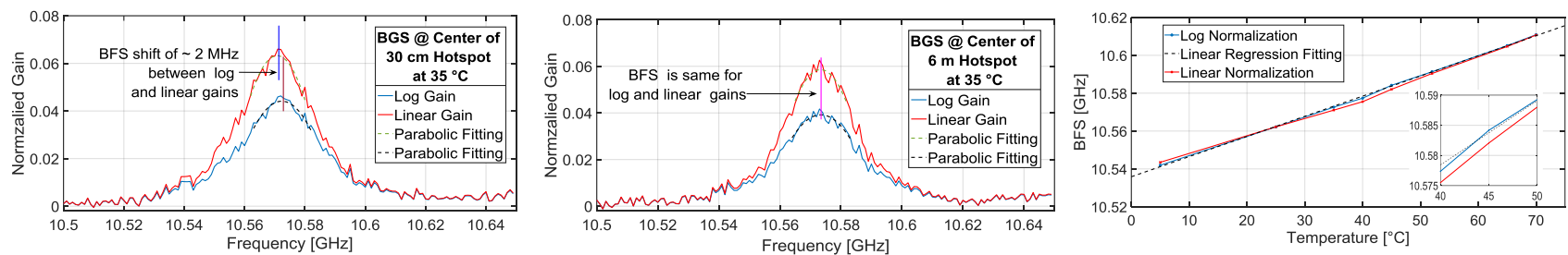

Figure 3. Left: The DPP-BOTDA processed BGS at the center of the $30 \mathrm{~cm}$ hotspot $\left(\right.$ at $\left.35^{\circ} \mathrm{C}\right)$ using the log normalized gain (blue) and linearly normalized gain (red), exhibiting a noticeable BFS difference; Middle: The BGS at the center of the $6 \mathrm{~m}$ hotspot at the same temperature as to that of $30 \mathrm{~cm}$, BFSs of the two methods appear to be same; Right: BFS linearity of both processes showing log-gain as better fit to the temperature change from 5 to $70{ }^{\circ} \mathrm{C}$, the zoomed section further clarifies.

Figure 3 on the left shows BGSs at temperature of $35^{\circ} \mathrm{C}$ for the two methods at center of the $30 \mathrm{~cm}$ hotspot, where BFS values (obtained by parabolic fitting) differ. At the center of the $6 \mathrm{~m}$ hotspot (middle pane), the two BFS values coincide. 
It is further experimentally observed in the right pane that the log-normalized BFS values for the $30 \mathrm{~cm}$ segment exhibit better linear fit to the temperature changes.

\section{CONCLUSIONS}

The subtraction of linearly normalized gain for the BFS estimations in DPP-BOTDA produces results that deviate from those obtained from the subtraction of log normalized gains by as much as $2 \mathrm{MHz}$ for hotspot sizes of the order of the spatial resolution of the technique ( $30 \mathrm{~cm}$ when using pump pulses widths of 40 and $43 \mathrm{~ns}$ ). For much longer hotspots, there are only small deviations, which occur only towards the end of the segment. Experimental results indicate that log normalized gain (resulted from subtraction of linear gains) method of gain subtraction represents true results in all conditions.

\section{ACKNOWLEDGEMENT}

The work of Hari Bhatta was performed in the framework of ITN-FINESSE, funded by the European Union's Horizon 2020 research and innovation program under the Marie Sklodowska-Curie Action grant agreement $n^{\circ} 722509$.

\section{REFERENCES}

[1] Bai, Q. et al., "Recent Advances in Brillouin Optical Time Domain Reflectometry," Sensors, 19(8), 1862, doi:10.3390/s19081862 (2019)

[2] Krohn, D. A., and MacDougall, T. W., [Fiber optic sensors: fundamentals and applications], SPIE Press, (2015).

[3] Yang, Z. et al., "Design rules for optimizing unipolar coded Brillouin optical time-domain analyzers," Opt. Express 26, 16505-16523 (2018).

[4] Motil, A., Bergman, A., and Tur, M., "State of the art of Brillouin fiber-optic distributed sensing," Opt. Laser Technol., vol. 78, pp. 81-103, (2016).

[5] Barrias, A., Casas, J. R., and Villalba, S., "A Review of Distributed Optical Fiber Sensors for Civil Engineering Applications," Sensors, 16(5), 748, doi: 10.3390/s16050748 (2016).

[6] Feng, C., Kadum, J. E., and Schneider T., "The State-of-the-Art of Brillouin Distributed Fiber Sensing", IntechOpen, DOI: 10.5772/intechopen.84684 (2019)

[7] Soto, M. A., and Thévenaz, L., "Modeling and evaluating the performance of Brillouin distributed optical fiber sensors," Opt. Express 21, 31347-31366 (2013)

[8] Li, W. et al., "Differential pulse-width pair BOTDA for high spatial resolution sensing," Opt. Express 16, 2161621625 (2008)

[9] Motil, A. et al., "Gain dependence of the linewidth of Brillouin amplification in optical fibers," Opt. Express 22, 27535-27541 (2014)

[10] Alem, M., Soto, M.A., Tur, M., Thévenaz, L., "Analytical expression and experimental validation of the Brillouin gain spectral broadening at any sensing spatial resolution," Proc. SPIE 10323, 25th International Conference on Optical Fiber Sensors, 103239J (2017) 